\title{
Small bowel metastasis from pulmonary rhabdomyosarcoma causing intussusception: a case report
}

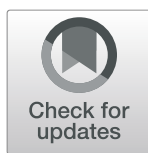

Ke-kang Sun and Xiao-jun Shen *id

\begin{abstract}
Background: Rhabdomyosarcoma (RMS), especially primary pulmonary RMS, is an extremely rare type of soft tissue sarcoma in adults. Small bowel is an uncommon site for metastases.

Case presentation: This report described an unusual case of jejunum metastasis from primary pulmonary RMS causing intussusception in a 75-year-old man. The patient consulted for 2 weeks of continuous dyspnea. Chest computed tomography (CT) demonstrated a large mass involving the left lower lobe. Transthoracic biopsy confirmed the existence of pleomorphic RMS. Immunohistochemical studies showed positive findings about desmin and MyoD1. The results of gastroscopy, colonoscopy and abdominal CT were all negative. Positron emission tomography/CT demonstrated a fluorodeoxyglucose-reactive large lesion in the left lower lobe without metastatic lesions. The patient received synchronous chemoradiotherapy. After 9 months, the patient presented with intermittent upper abdominal pain with nausea and vomiting. CT showed small bowel dilatation secondary to intussusception. The patient subsequently received laparotomy, and the intussuscepted small bowel segment was resected. Histological examination revealed pleomorphic RMS involving the mucosa, submucosa, and muscular tissues.
\end{abstract}

Conclusions: RMS is highly aggressive and metastatic. The metastatic disease can rapidly progress to cause subsequent complications. The possibility of small bowel metastasis should be considered, although it is extremely rare.

Keywords: Rhabdomyosarcoma, Small bowel metastasis, Intussusception, Case report

\section{Background}

Rhabdomyosarcoma (RMS) is a malignant skeletal muscle neoplasm. RMS is a common soft tissue sarcoma that occurs during childhood and adolescence, and it exists in the first 2 decades of life. By contrast, RMS is remarkably rare in adults. RMS accounts for $<1 \%$ of adult malignancies and $<3 \%$ of adult soft tissue sarcomas [1]. In addition, pleomorphic RMS is overrepresented in adult series. RMS in adults has a poor outcome compared with that in young individuals regardless of the implementation of multimodal therapies [2,3]. RMS is commonly found in head and neck, genitourinary tract, and retroperitoneum [4]. Primary pulmonary rhabdomyosarcoma (PPR) is

\footnotetext{
*Correspondence: shenxiaojun720128@126.com

Clinical Medical College of Jiangsu University, Department of Gastrointestinal

Surgery, Kunshan First People's Hospital affiliated to Jiangsu University,

Jiangsu, Kunshan 215300, China
}

remarkably rare, and limited information about its clinicopathological features is reported in the literature [5-8]. RMS is highly invasive and metastatic. Metastases are mostly found in bones, bone marrows, lungs, or lymph nodes [9]. RMS metastases on the small bowel are few and far between. Herein, we present a case of jejunum metastasis due to PPR, causing intussusception in a 75-year-old man.

\section{Case presentation}

The subject was a 75-year-old man with chronic obstructive pulmonary disease for more than 20 years. The patient was a nonsmoker and had no history of other remarkable illnesses. The patient consulted due to 2 weeks of continuous dyspnea without fever, cough, hemoptysis, or chest pain. Lung auscultation revealed no wheezing sounds. Laboratory examinations, including 
complete blood count, electrolytes, renal function, liver function, and urinalysis, were negative. Chest computed tomography (CT) confirmed the presence of a large mass lesion of $5.8 \mathrm{~cm} \times 4.4 \mathrm{~cm}$ that involves the left lower lobe (Fig. 1a and b). No lymphadenopathy or pleural effusion was observed. No mucosal lesions were identified through flexible fiberoptic bronchoscopy. CT-guided percutaneous transthoracic biopsy was conducted. Histological examination showed numerous compactly clustered small malignant cells with pleomorphism. A high mitotic rate was

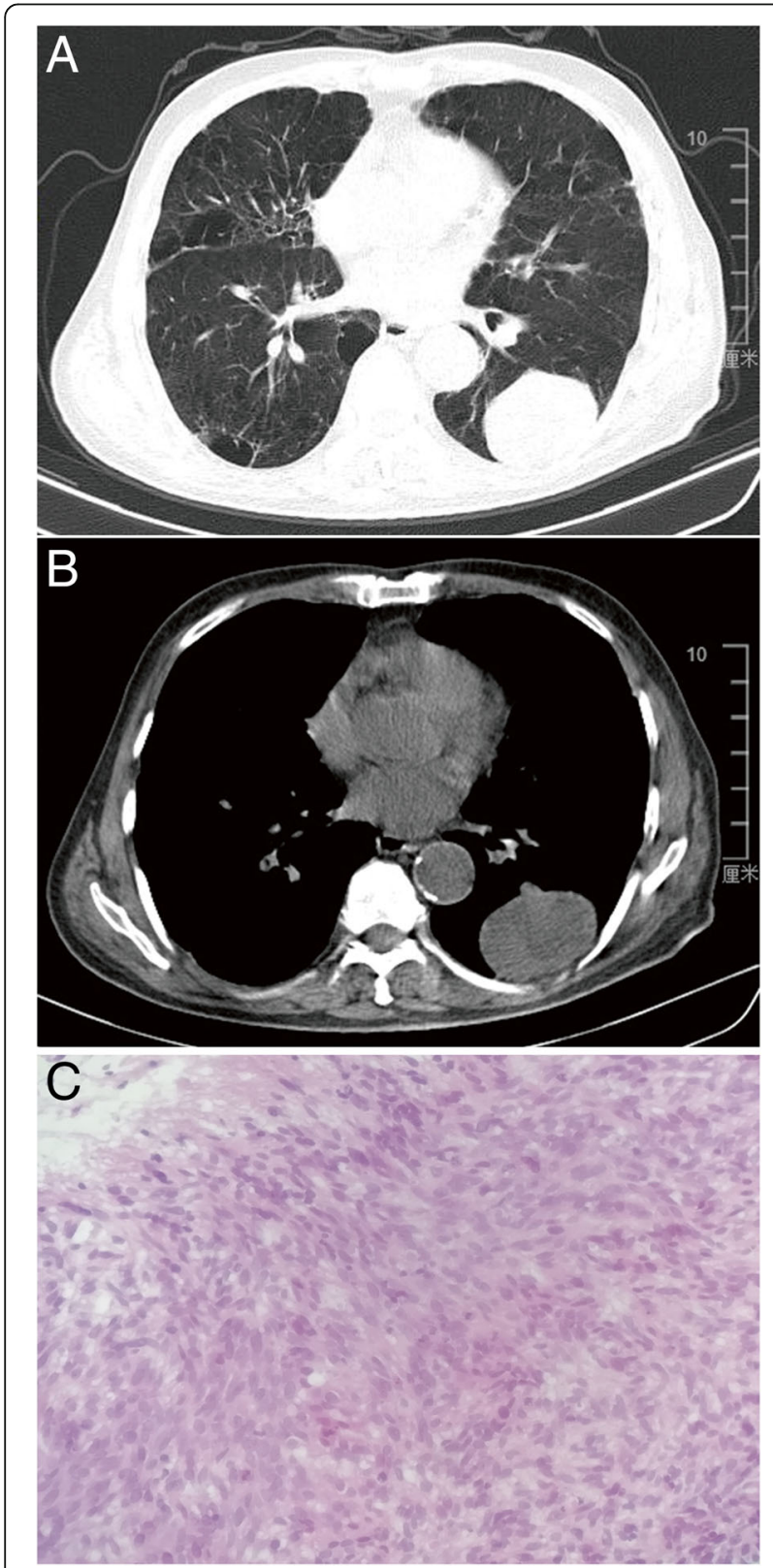

Fig. 1 CT scan shows a large solid mass in the left lower lobe: (a) Pulmonary window. b Mediastinal window. c CT-guided percutaneous transthoracic biopsy showing numerous compactly clustered small malignant cells with pleomorphism (hematoxylin and eosin stain, $\times 100$ ) observed (Fig. 1c). Several rhabdomyoblasts were observed in the partial area. Immunohistochemistry showed that the cells were positive for desmin and MyoD1 and negative for c-KIT and S-100 protein, which were consistent with the diagnosis of pleomorphic RMS. Extension study was conducted through gastroscopy, colonoscopy, abdominal CT, and bone scan. The results were negative. The patient was referred for entire body fluorodeoxyglucose positron emission tomography (PET)/CT to exclude metastatic tumors. The result demonstrated a fluorodeoxyglucose-reactive large lesion in the left lower lobe with a maximum standardized uptake value of 12.8 without metastatic lesions. The patient could not bear surgical resection because of poor lung function. The patient received two cycles of vincristine/cyclophosphamide/actinomycin D chemotherapy combined with 40 Gy of intensity-modulated radiation therapy (IMRT). The patient opted to stop chemotherapy because of general weakness. After 6 months, abdominal ultrasound revealed large mass lesions in bilateral adrenal glands, which are suspected for metastasis, and the patient discontinued the therapy.

After 9 months, the patient was referred again to our hospital because of intermittent upper abdominal pain

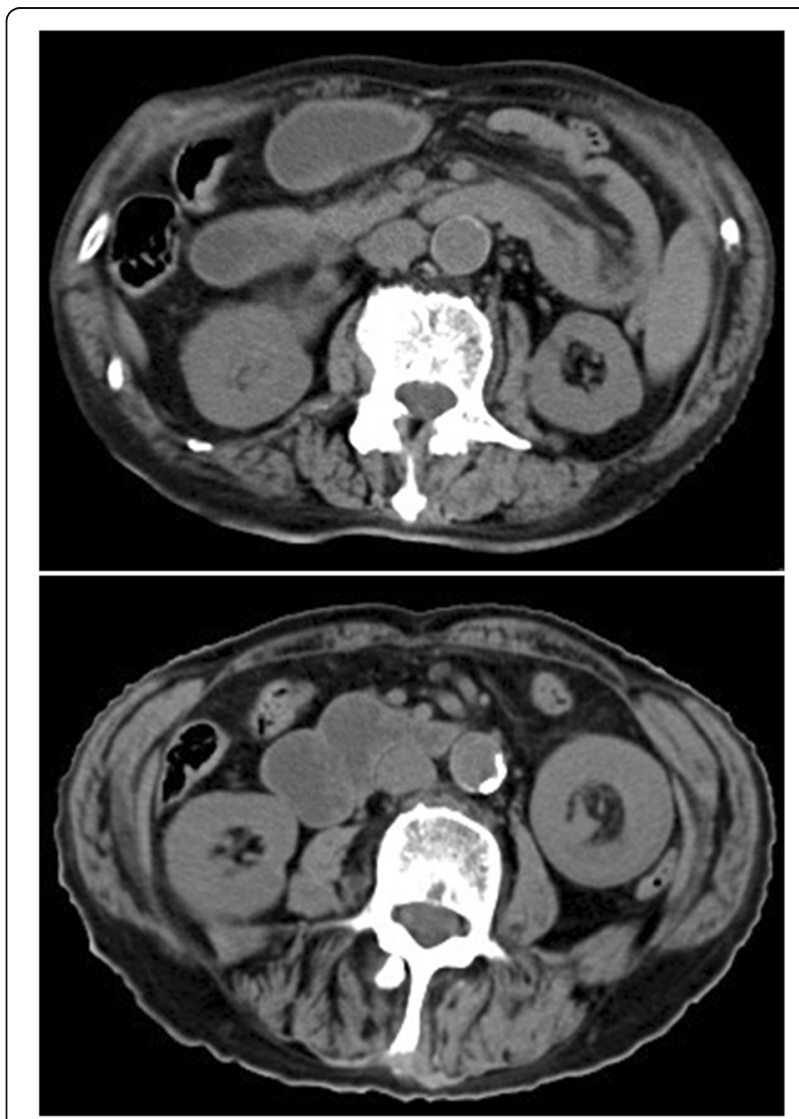

Fig. $2 \mathrm{CT}$ shows small bowel dilatation secondary to entero-enteric intussusception 
with nausea and vomiting. Physical examination showed that a large mass was palpable in the left abdomen. Bowel sounds were active. Laboratory data showed $2.1 \mathrm{mmol} / \mathrm{L}$ serum potassium. Abdominal CT showed small bowel dilatation secondary to entero-enteric intussusception (Fig. 2). The patient was given gastrointestinal decompression, potassium supplementation, and nutritional support treatment. Subsequently, laparotomy was performed, and a $15 \mathrm{~cm}$ segment of non-gangrenous intussusception was found in the jejunum with a $3 \mathrm{~cm}$ tumor forming the lead point of intussusception (Fig. 3a and b). No enlarged lymph nodes were observed in the mesentery of the affected small bowel. No other lesions were detected in the small bowel and colon. The affected segment of the small bowel was resected with end-to-end small bowel stapled anastomosis. Postoperative period was generally predictable. The histological examination of the surgical specimen revealed a pleomorphism malignant cell tumor that involved the mucosa, submucosa, and muscular tissues (Fig. 3c and d). Immunohistochemical studies were positive for desmin and MyoD1. These findings demonstrated that RMS originated in the lung. To date, the patient survived for 1 year after he was initially diagnosed and is currently under a good general condition.

\section{Discussion and conclusions}

RMS is a common soft tissue sarcoma in the pediatric population. RMS is initially believed to originate from skeletal muscles. However, RMS is currently known to arise from early rhabdomyoblasts that can be found in various locations throughout the body with varying degrees of differentiation [10]. RMS that originates from the lung is extremely rare. Limited information is reported about the clinicopathological features of adults with RMS in the literature. To our knowledge, only a limited number of studies focus on disease processes, diagnosis or treatment in children with rhabdomyosarcomas. Older patient age at presentation, unfavorable sites of the primary tumor, the size of the primary tumor, an alveolar subtype, and the presence of regional lymph node metastasis have been recognized as prognostic factors for rhabdomyosarcoma [11]. PPR should be diagnosed only after the possibility of metastatic RMS is excluded by extensive clinical examinations. In this case, the patient could not receive contrast-enhanced CT examination because of severe allergy to iodinated contrast medium. Extension study was achieved through gastroscopy, colonoscopy, abdominal CT, bone scan, and $\mathrm{PET} / \mathrm{CT}$ to exclude metastatic tumors. The results were negative.

Approximately 20\% of patients have distant metastases upon presentation, which is commonly found in lungs, bones, bone marrows, lymph nodes, and rare metastatic sites [11-13]. Metastases to the small bowel are extremely rare. This condition may be due to dense lymphatic tissues in the intestines, which contain a large
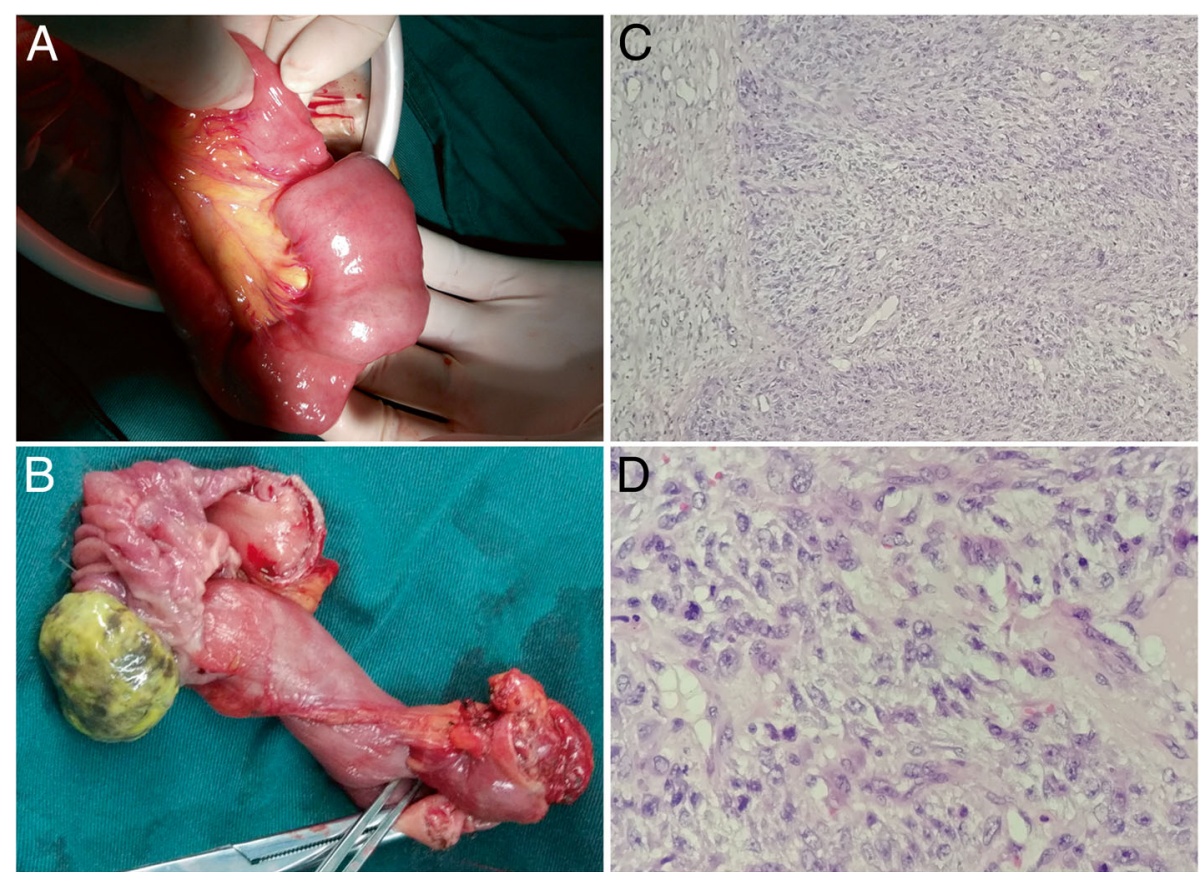

Fig. 3 a Intra-operative findings of jejuno-jejunal intussusception. b Intraluminal mass forming the pathologic lead point. c Histological examination of the surgical specimen with a pleomorphism malignant cell tumor that involves the mucosal, submucosal, and muscular tissues (hematoxylin and eosin stain, $\times 100$ ). $\mathbf{d}$ Several mitoses are observed in one high-power field (hematoxylin and eosin stain, $\times 400$ ) 
amount of $\mathrm{T}$ lymphocytes. The general route of small bowel metastasis is believed to be hematogenous in the spinal vein, retroperitoneal mediastinum, and lymphatic metastasis of the mesentery. Most small bowel metastases are caused by primary tumors, such as malignant melanoma, colon cancer, and cervical cancer [14]. The prognosis of patients with small bowel metastases is fairly poor, and most of them are found in serious complications, such as perforation, obstruction, and hemorrhage. Intussusception is rare in adults, and most cases are due to a pathologic lead point within the bowel and are malignant in more than $50 \%$ of cases, which require surgery and resection of the affected bowel segment. The common cause of malignant lesions is mainly primary tumor, and limited reports are available about intussusception secondary to small bowel metastases $[15,16]$.

The incidence of small bowel metastases is low, and misdiagnosis and missed diagnosis easily occur due to nonspecific symptoms. We reported the second case of small bowel intussusception caused by PPR metastasis, suggesting that the possibility of metastasis should be considered regardless of the rarity or site location [17]. Intussusception in adults should be investigated. In exploratory laparotomy, the intestinal tube should be thoroughly examined in terms of the presence of neoplastic lesions.

\section{Abbreviations}

CT: Computed tomography; IMRT: Intensity-modulated radiation therapy; PET: Positron emission tomography; PPR: Primary pulmonary rhabdomyosarcoma; RMS: Rhabdomyosarcoma

\section{Acknowledgments}

We would like to thank colleagues at the department of radiology and pathology in our hospital for providing the data.

\section{Funding}

This research did not receive any specific grant from funding agencies in the public, commercial, or not-for-profit sectors.

Availability of data and materials

All data generated or analyzed during this study are included in this published article.

\section{Authors' contributions \\ XJ S supervised all aspects of this research, and KK S prepared the manuscript. Both authors have read and approved the manuscript.}

\section{Ethics approval and consent to participate}

The present study was undertaken under the guidelines of the Ethics Committee of Jiangsu University. The authors have declared no ethical conflicts.

\section{Consent for publication}

Written informed consent of publication was obtained from the patient in the study.

\section{Competing interests}

The authors declare that they have no competing interests.

\section{Publisher's Note}

Springer Nature remains neutral with regard to jurisdictional claims in published maps and institutional affiliations.

Received: 6 November 2018 Accepted: 24 April 2019

Published online: 10 May 2019

\section{References}

1. Nascimento AF. Rhabdomyosarcomas in adults: classification and differential diagnosis. Diagnostic Histopathology. 2008;14(11):538-45.

2. Fischer TD, Gaitonde SG, Bandera BC, et al. Pediatric-protocol of multimodal therapy is associated with improved survival in AYAs and adults with rhabdomyosarcoma. Surgery. 2018;163(2):324-9.

3. Dumont SN, Araujo DM, Munsell MF, et al. Management and outcome of 239 adolescent and adult rhabdomyosarcoma patients. Cancer Med. 2013;2(4):553-63.

4. Wasti AT, Mandeville H, Gatz S, et al. Rhabdomyosarcoma. Paediatr Child Health. 2018;28(4):157-63.

5. Choi JS, Choi JS, Kim EJ. Primary pulmonary rhabdomyosarcoma in an adult with neurofibromatosis-1. Ann Thorac Surg. 2009;88(4):1356-8.

6. Gupta A, Sharma MC, Kochupillai $V$, et al. Primary pulmonary rhabdomyosarcoma in adults: case report and review of literature. Clin Lung Cancer. 2007;8(6):389-91.

7. Gray JA, Nguyen GK. Primary pulmonary rhabdomyosarcoma diagnosed by fine-needle aspiration cytology. Diagn Cytopathol. 2003;29(3):181-2.

8. Comin CE, Santucci M, Novelli L, et al. Primary pulmonary rhabdomyosarcoma: report of a case in an adult and review of the literature. Ultrastruct Pathol. 2001;25(3):269-73.

9. Ferrari A, Dileo P, Casanova M, et al. Rhabdomyosarcoma in adults. A retrospective analysis of 171 patients treated at a single institution. Cancer. 2003;98(3):571-80.

10. Parham DM, Ellison DA. Rhabdomyosarcomas in adults and children: an update. Arch Pathol Lab Med. 2006:130:1454-65.

11. Kim JR, Yoon HM, Koh KN, et al. Rhabdomyosarcoma in children and adolescents: patterns and risk factors of distant metastasis. AJR Am J Roentgenol. 2017;209(2):409-16.

12. Ismail $E$, Issam L, Hamid M. Pituitary metastasis of rhabdomyosarcoma: a case report and review of the literature. J Med Case Rep. 2014;8:144.

13. Elomrani F, Touri S, Ouziane I, et al. Orbital rhabdomyosarcoma with skin metastasis: a case report. BMC Res Notes. 2014;7:670.

14. Marko L, Veronique B, Zoran K. Melanoma of the small intestine. The Lancet Oncology. 2009;10(5):516-21.

15. Kim HS, Shin JW, Kim GY, et al. Metastasis of hepatocellular carcinoma to the small bowel manifested by intussusception. World J Gastroenterol. 2006;12(12):1969-71

16. Tan QT, Teo JY, Ahmed SS, et al. A case of small bowel metastasis from spinal Ewing sarcoma causing intussusception in an adult female. World J Surg Oncol. 2016:14:109.

17. Xi S, Tong W. Pleomorphic rhabdomyosarcoma metastasis to small intestine causing intussusception: a case report. Medicine (Baltimore) 2018; 97(51): e13648.

Ready to submit your research? Choose BMC and benefit from:

- fast, convenient online submission

- thorough peer review by experienced researchers in your field

- rapid publication on acceptance

- support for research data, including large and complex data types

- gold Open Access which fosters wider collaboration and increased citations

- maximum visibility for your research: over $100 \mathrm{M}$ website views per year

At BMC, research is always in progress.

Learn more biomedcentral.com/submissions 